\title{
A REFINED MIXED FINITE ELEMENT METHOD FOR STATIONARY NAVIER - STOKES EQUATIONS WITH MIXED BOUNDARY CONDITIONS USING LAGRANGE MULTIPLIERS
}

\author{
S. NICAISE ${ }^{1}$, L. PAQUET ${ }^{2}$, AND RAFILIPOJAONA $^{3}$
}

\begin{abstract}
This paper is concerned with a dual mixed formulation of the Navier Stokes system in a polygonal domain of the plane with mixed boundary conditions and its numerical approximation. The Neumann boundary condition is imposed using a Lagrange multiplier corresponding to the velocity field. Moreover, the strain tensor and the antisymmetric gradient tensor (vorticity), quantities of practical interest, are introduced as new unknowns. The problem is then approximated by a mixed finite element method. Quasi-optimal error estimates are finally obtained using refined meshes near singular corners.
\end{abstract}

2000 Mathematics Subject Classification: 65N15, 65N30.

Keywords: Navier — Stokes system, mixed FEM, singularities.

\section{Introduction}

Any solution of the Navier — Stokes equations in polygonal domains has in general corner singularities $[16,24,36]$. Hence standard numerical methods lose accuracy on quasi-uniform meshes, and locally refined meshes are necessary to restore the optimal order of convergence. Standard finite element methods for second order elliptic operators, the Stokes or the Navier - Stokes system with corner singularities (and mixed boundary conditions) have been analyzed in $[2,3,5,6,8,9,18,36,37]$, where it has been shown that the use of appropriate refined meshes near the singular points allows to restore the optimal order of convergence. Mixed methods for the Stokes and Navier - Stokes with Dirichlet boundary conditions were initiated in $[20,22]$. Similarly, a mixed method for the Boussinesq equations (coupling between the Navier - Stokes equations and the heat equation) with Dirichlet boundary conditions on the velocity field and corner singularities was analyzed in [24] (some geometrical restrictions were imposed due to the mixed boundary conditions on temperature). In these papers, only $\nabla u$ was introduced as a new unknown and, therefore, the natural Neumann boundary conditions cannot be treated. Mixed methods for the linear elasticity with mixed boundary conditions (with or without singularities) were considered in [20,22,25], where the authors introduced the strain tensor and the vorticity as new unknowns. Finally, let us recall

\footnotetext{
${ }^{1}$ Université de Valenciennes et du Hainaut Cambrésis, LAMAV, ISTV, F-59313 - Valenciennes Cedex 9, France. E-mail: snicaise@univ-valenciennes.fr, http://www.univ-valenciennes.fr/macs/nicaise

${ }^{2}$ Université de Valenciennes et du Hainaut Cambrésis, LAMAV, ISTV, F-59313 - Valenciennes Cedex 9, France. E-mail: Luc.Paquet@univ-valenciennes.fr

${ }^{3}$ Universté de Fianarantsoa, Faculté des Sciences, B.P. 1486, Fianarantsoa 301, Madagascar. E-mail: rafilipo@wanadoo.mg
} 
that the interests of mixed methods are twofold. First, the Neumann boundary condition becomes an essential boundary condition and, second, its discretization gives directly approximated strain tensor and vorticity without any postprocessing. Nevertheless in standard dual mixed finite element methods, only homogeneous Neumann boundary conditions can be treated. Nonhomogeneous boundary conditions can be managed if we introduce, as a new unknown, a Lagrange multiplier on the part of the boundary where Neumann boundary conditions are imposed. This was done successfully by Babuska and Gatica for the Laplace equation in [7] and by the authors for the Lamé system in [35].

Our goal here is to consider the stationary Navier - Stokes equations with mixed boundary conditions (the Dirichlet boundary condition on a part of the boundary $\Gamma_{D}$ and the Neumann one on the remaining part $\Gamma_{N}$ ) in a two-dimensional polygonal domain and to approximate them by a mixed finite element method. Since the Neumann boundary condition is imposed on a part of the boundary, our method uses the new unknowns of physical meaning, which are the rate of the strain tensor $\varepsilon(u)=\left(\nabla u+(\nabla u)^{\top}\right) / 2$ and the vorticity $\omega(u)=\left(\nabla u-(\nabla u)^{\top}\right) / 2, u$ being the velocity field. As said before, we further introduce the Lagrange multiplier $\xi=u_{\mid \Gamma_{N}}$. The poor regularity of any solution forces us to use appropriate Banach spaces and then introduce an appropriate mixed formulation of the problem. We further establish equivalence between the classical variational formulation of the problem and this new mixed formulation. We next consider some discretization of our mixed formulation by using some mixed finite elements developed by Farhloul and Fortin [22]. Namely the approximation spaces are piecewise constant for the velocity, piecewise $P_{1}$ for the pressure, piecewise $\left[P_{1}\right]^{2}+$ curl of element bubbles for each line of the strain tensor, piecewise $\left[P_{1}\right]^{2 \times 2}$ for the vorticity, and piecewise $P_{1}$ for the Lagrange multiplier. We then prove that the discrete mixed formulation has at least one solution near any nonsingular solution of the Navier - Stokes equations. Furthermore, using appropriate refined meshes near the singular points we show a quasi-optimal error estimate.

Our approach then combines some ideas from $[20,22,25]$ developed for the linear elasticity with the techniques from $[20,22,24]$ used for the Navier - Stokes equations and with the one from $[7,35]$.

Let us mention that in [11] numerical experiments were performed for the hybrid formulation of the same mixed method as here but concerning the Lamé system in an $L$-shaped domain for large Lamé coefficients $\lambda$. The convergence rates of errors on uniform meshes and on refined meshes constructed using Raugel's procedure (see below) were compared. These experiments suggest that the convergence rates of errors should have the same behavior for the Stokes equations (because as the Lamé coefficient $\lambda$ becomes large, the Lamé system tends to the Stokes one). Of course, for the Navier - Stokes equations, we have an additional difficulty due to the nonlinear convection term, but we think that we could resolve it by using a Newton - Galerkin scheme as in [21] although in that paper the mixed method therein considers rather the pressure, the velocity and its gradient as relevant unknowns. We plan to perform such numerical experiments in the future.

This paper is organized as follows. In Section 2, we derive incompressible Navier - Stokes equations with mixed boundary conditions, introduce their classical variational formulation, and recall some regularity results in weighted Sobolev spaces that will be useful for our further analysis. We further give our new mixed formulation and show its equivalence to the variational formulation. In Section 3, we give some useful properties of linear operators in some functional spaces related to nonsingular solutions. In Section 4, we describe and analyze the discretization of the problem. Finally, Section 5 is devoted to the proof of the quasi-optimal error estimate. 


\section{Mixed formulation of the Navier - Stokes equations with Dirichlet — Neumann boundary conditions}

Let $\Omega$ be a plane domain with a polygonal boundary. More precisely, we assume that $\Omega$ is a simply connected domain and that its boundary $\Gamma$ is the union of a finite number of linear segments $\bar{\Gamma}_{j}, 1 \leqslant j \leqslant n_{e}\left(\Gamma_{j}\right.$ is assumed to be an open segment). Let us further fix a partition $\{D, N\}$ of the set $\left\{j \in \mathbb{N} ; 1 \leqslant j \leqslant n_{e}\right\}$. In $\Omega$, we consider the following Navier Stokes equations:

$$
\left\{\begin{array}{l}
-2 \mu \operatorname{div} \varepsilon(u)+(u \cdot \nabla) u+\nabla p=f \quad \text { in } \Omega \\
\operatorname{div} u=0 \text { in } \Omega, \\
u=0 \text { on } \Gamma_{D}=\cup_{j \in D} \Gamma_{j}, \\
(2 \mu \varepsilon(u)-p \delta) \cdot n=g \text { on } \Gamma_{N}=\cup_{j \in N} \Gamma_{j}
\end{array}\right.
$$

where $\mu$ is the viscosity parameter, $u=\left(u_{1}, u_{2}\right)$ is the velocity field (throughout the paper, vector fields are written in line), $p$ is the pressure, $f$ is the density force supposed to be in $\left(L^{2}(\Omega)\right)^{2}, g$ is the surface force supposed to be in $\left(H^{1 / 2}\left(\Gamma_{N}\right)\right)^{2}, \delta$ is the identity matrix, $\varepsilon(u)=\left(\nabla u+(\nabla u)^{\top}\right) / 2$ is the strain tensor field, and $n$ is the unit outward normal vector field along the boundary of $\Omega$. For the tensor $\tau=\left(\tau_{i j}\right)_{1 \leqslant i, j \leqslant 2}$, the normal trace $\tau \cdot n$ is defined by $\tau \cdot n=\left(\sum_{j=1}^{2} \tau_{1 j} n_{j}, \sum_{j=1}^{2} \tau_{2 j} n_{j}\right)$. In the sequel, we make the natural assumption that $\Gamma_{D} \neq \varnothing$.

Throughout the paper, we will use the following notations. As usual, we denote by $L^{2}(\cdot)$ the Lebesgue space and by $H^{s}(\cdot), s \geqslant 0$ the standard Sobolev space. The usual norm and seminorm of $H^{s}(D)$ are denoted by $\|\cdot\|_{s, D}$ and $|\cdot|_{s, D}$. For shortness the $L^{2}(D)$-norm will be denoted by $\|\cdot\|_{D}$ and in the case of $D=\Omega$, we will drop the index $\Omega$. The inner product in $\left(L^{2}(\Omega)\right)^{2}$ will be written as $(\cdot, \cdot)$ and the duality pairing between $\left(H^{-1 / 2}(\Gamma)\right)^{2}$ and $\left(H^{1 / 2}(\Gamma)\right)^{2}$ will be denoted by $\langle\cdot, \cdot\rangle_{\Gamma}$. If $\sigma=\left(\sigma_{i j}\right), \tau=\left(\tau_{i j}\right) \in\left(L^{2}(\Omega)\right)^{2 \times 2}$, then we denote by

$$
\sigma: \tau=\sum_{i, j} \sigma_{i j} \tau_{i j}, \quad(\sigma, \tau)=\int_{\Omega} \sigma: \tau d x
$$

For any $q>1$, we recall that

$$
W_{q}(\operatorname{div} ; \Omega)=\left\{\left(v_{1}, v_{2}\right) \in\left(L^{q}(\Omega)\right)^{2}: \operatorname{div} v=\frac{\partial v_{1}}{\partial x_{1}}+\frac{\partial v_{2}}{\partial x_{2}} \in L^{q}(\Omega)\right\} .
$$

Moreover, $\left(W_{q}(\operatorname{div} ; \Omega)\right)^{2}$ means the set of matrix valued functions such that each line is in $W_{q}(\operatorname{div} ; \Omega)$.

For $\tau=\left(\tau_{i j}\right) \in\left(W_{q}(\operatorname{div} ; \Omega)\right)^{2}$, for some $q>1$, we denote by

$$
\operatorname{div} \tau=\left(\frac{\partial \tau_{11}}{\partial x_{1}}+\frac{\partial \tau_{12}}{\partial x_{2}}, \frac{\partial \tau_{21}}{\partial x_{1}}+\frac{\partial \tau_{22}}{\partial x_{2}}\right), \quad \text { as }(\tau)=\tau_{21}-\tau_{12} .
$$

For $v=\left(v_{1}, v_{2}\right) \in\left(W^{1, p}(\Omega)\right)^{2}$ for some $p>1$, we recall that

$$
\operatorname{curl} v=\frac{\partial v_{2}}{\partial x_{1}}-\frac{\partial v_{1}}{\partial x_{2}}
$$

The variational formulation of problem (2.1) is quite standard [36]: introduce the space

$$
H_{0, \Gamma_{D}}^{1}(\Omega)=\left\{u \in H^{1}(\Omega) ; u_{\mid \Gamma_{D}}=0\right\} .
$$


Set for shorthness $W=\left(H_{0, \Gamma_{D}}^{1}(\Omega)\right)^{2}$. Then we are looking for a pair $(u, p) \in W \times L^{2}(\Omega)$ solution of

$$
\begin{gathered}
2 \mu \int_{\Omega} \varepsilon(u): \varepsilon(v) d x+\int_{\Omega}[(u \cdot \nabla) u] \cdot v d x-\int_{\Omega} p \operatorname{div} v d x=\int_{\Omega} f \cdot v d x+\int_{\Gamma_{N}} g \cdot v d s(x), \quad \forall v \in W, \\
\int_{\Omega} q \operatorname{div} u d x=0, \quad \forall q \in L^{2}(\Omega) .
\end{gathered}
$$

This problem has at least one solution $(u, p)$ (not necessarily unique). Orlt and Sändig in [36] have proved that if $f$ and $g$ are sufficiently small, namely

$$
\left(\|f\|_{W^{*}}^{2}+\|g\|_{W^{*}}^{2}\right)^{1 / 2}<\alpha^{* 2} /(4 \gamma),
$$

then problem $(2.3),(2.4)$ has a unique solution $(u, p) \in W \times L^{2}(\Omega)$ in the open ball of center 0 and radius $\alpha^{*} /(2 \gamma)$ in $W$. In $(2.5), \alpha^{*}$ denotes the coerciveness constant on $W$ of the quadratic form

$$
W \rightarrow \mathbb{R}_{+}: w \mapsto \int_{\Omega}|\varepsilon(w)|^{2} d x
$$

and $\gamma$ is the norm of the trilinear form

$$
\left[H^{1}(\Omega)^{2}\right]^{3} \rightarrow \mathbb{R}:(u, v, w) \mapsto \int_{\Omega}[(u \cdot \nabla) v] \cdot w d x
$$

Here we shall not necessarily suppose that (2.5) holds.

Before going on let us present some results on regularity for a solution of problem (2.3), (2.4). By a quite standard bootstrap argument, this regularity is related to the singularities of the solution of the Stokes problem with mixed boundary conditions in $\Omega$. Here we give results on regularity for weighted Sobolev spaces which we need for the use of refined finite element methods. We first recall some notations of the results on regularity for this problem obtained in $[14,16,36]$. Let $\left\{S_{j}\right\}=\bar{\Gamma}_{j} \cap \bar{\Gamma}_{j+1}, j=1, \ldots, n_{e}$, be the vertices of $\Omega$ and let $\omega_{j}$ denote the interior angle of $\Omega$ at $S_{j}$. Then the singular exponents of the Stokes problem near $S_{j}$ are the roots $\lambda \in \mathbb{C} \backslash\{0\}$ of (see $[14,16,36]$ for more details):

$$
\begin{gathered}
\sin ^{2}\left(\lambda \omega_{j}\right)-\lambda^{2} \sin ^{2} \omega_{j}=0 \text { if } \Gamma_{j} \cup \Gamma_{j+1} \subset \Gamma_{D} \text { or } \Gamma_{N}, \\
\cos ^{2}\left(\lambda \omega_{j}\right)-\lambda^{2} \sin ^{2} \omega_{j}=0 \text { otherwise } .
\end{gathered}
$$

Let us set $\Lambda_{S, j}=\{\lambda ; \lambda$ is a solution of (2.6) or (2.7) and $\operatorname{Re} \lambda \in] 0,1[\}$ and $\xi_{S}\left(\omega_{j}\right)=$ $\min \left\{\operatorname{Re} \lambda ; \lambda \in \Lambda_{S, j}\right\}$ if $\Lambda_{S, j}$ is not empty, otherwise we take $\xi_{S}\left(\omega_{j}\right)=0$.

We further introduce the following weighted Sobolev spaces (see, e.g., [28]): for any positive integer $k$ and any nonnegative real number $\alpha$, we define

$$
H^{k, \alpha}(\Omega)=\left\{v \in H^{k-1}(\Omega) ; \quad r^{\alpha} D^{\beta} v \in L^{2}(\Omega), \quad \forall|\beta|=k\right\},
$$

which is a Hilbert space for the norm $\|v\|_{k, \alpha, \Omega}=\left(\|v\|_{k-1, \Omega}^{2}+|v|_{k, \alpha, \Omega}^{2}\right)^{1 / 2}$, where the seminorm $|\cdot|_{k, \alpha, \Omega}$ is defined by $|v|_{k, \alpha, \Omega}=\left(\sum_{|\beta|=k}\left\|r^{\alpha} D^{\beta} v\right\|_{0, \Omega}^{2}\right)^{1 / 2}, r(x)$ being the distance from $x$ to the vertices of $\Omega$.

Now we are able to present the following result on regularity which is a particular case of $[24$, Theorem 2.1]. 
Theorem 2.1. Let $(u, p) \in W \times L^{2}(\Omega)$ be a solution of $(2.3)$, (2.4) with $f \in\left(L^{2}(\Omega)\right)^{2}$ and $g \in\left(H^{1 / 2}\left(\Gamma_{N}\right)\right)^{2}$. Then

$$
(u, p) \in\left[H^{2, \alpha}(\Omega)\right]^{2} \times H^{1, \alpha}(\Omega),
$$

for all nonnegative $\alpha$ such that $\alpha>1-\min _{j=1, \ldots, n_{e}} \xi_{S}\left(\omega_{j}\right)$.

Note further that $u$ satisfies

$$
\begin{gathered}
u \in(C(\bar{\Omega}))^{2}, \\
(u \cdot \nabla) u \in\left(L^{2}(\Omega)\right)^{2} .
\end{gathered}
$$

Let us now recall the Green formulas which we will need subsequently (for the second formula, see Lemma 2.2 of [26]).

Lemma 2.1. Let $\tau \in\left(W_{q}(\operatorname{div} ; \Omega)\right)^{2}$ and $v \in\left(W^{1, p}(\Omega)\right)^{2}$ with $1 / p+1 / q=1$, then

$$
(\varepsilon(v), \tau)=\langle\tau \cdot n, v\rangle_{\Gamma}-(\operatorname{div} \tau, v)-(\operatorname{as}(\tau), \operatorname{curl} v) / 2,
$$

where $\langle\cdot, \cdot\rangle_{\Gamma}$ means the duality pair between $H^{-1 / q, q}(\Gamma)$ and $H^{1 / q, p}(\Gamma)$.

Lemma 2.2. Let $v \in\left[L^{s}(\Omega)\right]^{2}$ such that $\operatorname{div} v \in L^{t}(\Omega)$. Let us suppose that $s>1$ and $t>1$ verify $1 / t<1 / s+1 / 2$. Then $v \cdot n \in W^{-1 / s, s}(\Gamma)$ with the following identity

$$
\langle v \cdot n, \phi\rangle=(v, \nabla \phi)+(\operatorname{div} v, \phi), \quad \forall \phi \in W^{1, s^{\prime}}(\Omega)
$$

where $s^{\prime}>1$ is the conjuguate of $s$, i.e., $1 / s+1 / s^{\prime}=1$ and $\langle\cdot, \cdot\rangle$ means the duality pair between $W^{-1 / s, s}(\Gamma)$ and $W^{1 / s, s^{\prime}}(\Gamma)$.

The dual mixed variational formulation requires the introduction of the following new unknowns: the strain tensor

$$
\sigma=2 \mu \varepsilon(u)
$$

the vorticity

$$
\omega=\left(\nabla u-(\nabla u)^{\top}\right) / 2=(\operatorname{curl} u) \chi / 2
$$

where $\chi=\left(\begin{array}{cc}0 & -1 \\ 1 & 0\end{array}\right)$, and finally the Lagrange multiplier

$$
\xi=-\left.u\right|_{\Gamma_{N}}
$$

Let us further introduce the spaces

$$
\begin{gathered}
\Sigma=\left\{\underline{\tau}=(\tau, q) \in\left[L^{r_{1}}(\Omega)\right]^{2 \times 2} \times L^{r_{1}}(\Omega): \operatorname{div}(\tau-q \delta) \in\left(L^{t}(\Omega)\right)^{2}\right\} \\
M=\left\{\underline{v}=(v, \eta, \varphi): v \in\left(L^{r_{2}}(\Omega)\right)^{2} ; \quad \eta \in\left[L^{r_{1}}(\Omega)\right]^{2 \times 2}: \eta+\eta^{\top}=0 ; \quad \varphi \in\left(H_{00}^{1 / 2}\left(\Gamma_{N}\right)\right)^{2}\right\},
\end{gathered}
$$

where we recall that $[28] H_{00}^{1 / 2}\left(\Gamma_{N}\right)=\left\{\left.v\right|_{\Gamma_{N}}: v \in H_{0, \Gamma_{D}}^{1}(\Omega)\right\}$. Let us also recall that $H^{-1 / 2}\left(\Gamma_{N}\right)$ is the dual of $H_{00}^{1 / 2}\left(\Gamma_{N}\right)$.

In (2.15) and (2.16), the parameters $r_{1}, r_{2}, t$ are such that

$$
2<r_{1}<\frac{2}{\alpha}, \quad \frac{1}{r_{1}}+\frac{2}{r_{2}}=1, \quad t=\frac{4}{2+\alpha},
$$

with $\alpha \in] 0,1]$ such that $\alpha>1-\min _{1 \leqslant j \leqslant n e} \xi_{S}\left(\omega_{j}\right)$.

With these new unknowns, we get 
Theorem 2.2. $(u, p) \in\left(H_{0, \Gamma_{D}}^{1}(\Omega)\right)^{2} \times L^{2}(\Omega)$ is a solution of the variational formulation (2.3), (2.4) if and only if $(\underline{\sigma}, \underline{u})=((\sigma, p),(u, \omega, \xi)) \in \Sigma \times M$ is a solution of the mixed variational formulation

$$
\begin{gathered}
\frac{1}{2 \mu}(\sigma, \tau)+(\tau, \omega)+(\operatorname{div}(\tau-q \delta), u)+\langle(\tau-q \delta) \cdot n, \xi\rangle=0, \quad \forall \underline{\tau}=(\tau, q) \in \Sigma, \\
-\frac{1}{2 \mu}(\sigma \cdot u, v)-(\omega \cdot u, v)+(\sigma, \eta)+(\operatorname{div}(\sigma-p \delta), v)+\langle(\sigma-p \delta) \cdot n, \varphi\rangle_{\Gamma_{N}}=-(f, v)+\langle g, \varphi\rangle_{\Gamma_{N}} \\
\forall \underline{v}=(v, \eta, \varphi) \in M
\end{gathered}
$$

with relations $(2.12)-(2.14)$.

Proof. Let us first check that the terms in (2.17) and (2.18) are well defined. First

$$
(\sigma, \tau)=\int_{\Omega} \sigma: \tau d x=\int_{\Omega} \sum_{i, j=1}^{2} \sigma_{i j} \tau_{i j} d x, \quad(\sigma, \eta)=\int_{\Omega} \sigma: \eta=\int_{\Omega} \sum_{i, j=1}^{2} \sigma_{i j} \eta_{i j} d x
$$

are well defined because $\sigma, \eta, \tau \in\left(L^{r_{1}}(\Omega)\right)^{2 \times 2}$ and $r_{1}>2$. On the other hand, the condition $r_{1}<2 / \alpha$ implies that $1 / t=(2+\alpha) / 4<1 / 2+1 /\left(2 r_{1}\right)=1-1 / r_{2}$ and then $1 / t+1 / r_{2}<1$. This last property implies that the two terms $(\operatorname{div}(\tau-q \delta), u)$ and $(\operatorname{div}(\sigma-p \delta), v)$ are well defined. The two nonlinear terms $(\sigma \cdot u, v)$ and $(\omega \cdot u, v)$ are also well defined because $1 / r_{1}+2 / r_{2}=1$.

For the term $\langle(\tau-q \delta) \cdot n, \xi\rangle_{\Gamma_{N}}$, by the definition of $\Sigma, \tau-q \delta \in\left(L^{r_{1}}(\Omega)\right)^{2 \times 2}$ and $\operatorname{div}(\tau-$ $q \delta) \in\left(L^{t}(\Omega)\right)^{2}$. Therefore, by Lemma $2.2,(\tau-q \delta) \cdot n \in\left(W^{-1 / r_{1}, r_{1}}\left(\Gamma_{N}\right)\right)^{2}$ with $1 / t<1 / r_{1}+$ $1 / 2$ and $s>2$. But the Sobolev embedding theorem yields $W^{-1 / r_{1}, r_{1}}\left(\Gamma_{N}\right) \hookrightarrow H^{-1 / 2}\left(\Gamma_{N}\right)$. Consequently, $\langle(\tau-q \delta) \cdot n, \xi\rangle_{\Gamma_{N}}$ is well defined. The term $\langle(\sigma-p \delta) \cdot n, \varphi\rangle_{\Gamma_{N}}$ is well defined in the same way.

Let us prove that (2.3), (2.4) imply (2.17), (2.18): Let $(u, p) \in\left(H_{0, \Gamma_{D}}^{1}(\Omega)\right)^{2} \times L^{2}(\Omega)$ be a solution of $(2.3),(2.4)$. Then we respectively define $\sigma, \omega$, and $\xi$ by $(2.12),(2.13)$ and $(2.14)$. The requested regularities for $\sigma, p, \operatorname{div}(\sigma-p \delta)$ and $\omega$ follow from Theorem 2.1 (see [26]). On the other hand, since $u \in\left(H_{0, \Gamma_{D}}^{1}(\Omega)\right)^{2}$, we directly obtain $\xi=-u_{\mid \Gamma_{N}} \in\left(H_{00}^{1 / 2}\left(\Gamma_{N}\right)\right)^{2}$. Therefore, we can conclude that $\underline{\sigma}=(\sigma, p) \in \Sigma$ and that $\underline{u}=(u, w, \xi) \in M$.

In view $(2.3)$ we have $(\sigma-p \delta, \varepsilon(v))+((u \cdot \nabla) u, v)=(f, v), \forall v \in(\mathcal{D}(\Omega))^{2}$. Using the Green formula $(2.11)$, this identity can be written as $-(\operatorname{div}(\sigma-p \delta), v)+((u \cdot \nabla) u, v)=(f, v), \forall v \in$ $(\mathcal{D}(\Omega))^{2}$. Owing to $(2.10)$, this last identity leads to $\operatorname{div}(\sigma-p \delta)=(u \cdot \nabla) u-f \in\left(L^{2}(\Omega)\right)^{2}$. This identity implies that

$$
(\operatorname{div}(\sigma-p \delta), v)=((u \cdot \nabla) u-f, v), \quad \forall v \in\left(H_{0, \Gamma_{D}}^{1}(\Omega)\right)^{2} .
$$

In view the Green formula (2.11), we obtain

$$
\langle(\sigma-p \delta) \cdot n, v\rangle_{\Gamma_{N}}=(\sigma-p \delta, \varepsilon(v))+((u \cdot \nabla) u, v)-(f, v), \quad \forall v \in\left(H_{0, \Gamma_{D}}^{1}(\Omega)\right)^{2} .
$$

By (2.3), the right-hand side of this identity is equal to $\langle g, v\rangle_{\Gamma_{N}}$ and consequently

$$
\langle(\sigma-p \delta) \cdot n, v\rangle_{\Gamma_{N}}=\langle g, v\rangle_{\Gamma_{N}}, \quad \forall v \in\left(H_{0, \Gamma_{D}}^{1}(\Omega)\right)^{2}
$$

By the definition of $H_{00}^{1 / 2}\left(\Gamma_{N}\right)$, we deduce that

$$
\langle(\sigma-p \delta) \cdot n, \varphi\rangle_{\Gamma_{N}}=\langle g, \varphi\rangle_{\Gamma_{N}}, \quad \forall \varphi \in\left(H_{00}^{1 / 2}\left(\Gamma_{N}\right)\right)^{2}
$$


Summing this identity with (2.19), we get

$$
\begin{gathered}
(\operatorname{div}(\sigma-p \delta), v)+\langle(\sigma-p \delta) \cdot n, \varphi\rangle_{\Gamma_{N}}=((u \cdot \nabla) u, v)-(f, v)+\langle g, \varphi\rangle_{\Gamma_{N}}, \\
\forall v \in\left(H_{0, \Gamma_{D}}^{1}(\Omega)\right)^{2}, \quad \forall \varphi \in\left(H_{00}^{1 / 2}\left(\Gamma_{N}\right)\right)^{2}
\end{gathered}
$$

or equivalently

$$
\begin{gathered}
-((u \cdot \nabla) \cdot u, v)+(\operatorname{div}(\sigma-p \delta), v)+<(\sigma-p \delta) \cdot n, \varphi>_{\Gamma_{N}}=-(f, v)+<g, \varphi>_{\Gamma_{N}}, \\
\forall v \in\left(H_{0, \Gamma_{D}}^{1}(\Omega)\right)^{2}, \quad \forall \varphi \in\left(H_{00}^{1 / 2}\left(\Gamma_{N}\right)\right)^{2} .
\end{gathered}
$$

But we readily check that $(\sigma, \eta)=0$ for all $\eta \in\left(L^{r_{1}}(\Omega)\right)^{2 \times 2}$ such that $\eta+\eta^{\top}=0$. Therefore, for such $\eta$ we get $((u \cdot \nabla) \cdot u, v)=(2 \mu)^{-1}(\sigma \cdot u, v)+(\omega \cdot u, v)$. This identity and (2.20) lead to $(2.18)$.

Now let us fix $\underline{\tau}=(\tau, q) \in \Sigma$. Then we clearly have

$$
\frac{1}{2 \mu}(\sigma, \tau)+(\tau, \omega)=(\varepsilon(u), \tau)+\frac{1}{2}\left(\nabla u-(\nabla u)^{\top}, \tau\right)=\frac{1}{2}\left(\nabla u-(\nabla u)^{\top}, \tau\right)+\frac{1}{2}\left(\nabla u-(\nabla u)^{\top}, \tau\right)=(\nabla u, \tau) .
$$

On the other hand, identity (2.4) is equivalent to $(\nabla u, q \delta)=0$. Combining with (2.21), we get $(2 \mu)^{-1}(\sigma, \tau)+(\tau, \omega)=(\nabla u, \tau-q \delta)$. Using Lemma 2.2, this last identity yields

$$
(2 \mu)^{-1}(\sigma, \tau)+(\tau, \omega)-\langle(\tau-q \delta) \cdot n, u\rangle_{\Gamma_{N}}+(\operatorname{div}(\tau-q \delta), u)=0
$$

Recalling that $u_{\mid \Gamma_{N}}=-\xi$, we have obtained (2.17).

Reciprocally, let us show that (2.17), (2.18) imply (2.3), (2.4). Let

$$
(\underline{\sigma}, \underline{u})=((\sigma, p),(u, w, \xi)) \in \Sigma \times M
$$

be a solution of $(2.17),(2.18)$.

Let us first take $\underline{\tau}=(\tau, 0)$ with $\tau \in(\mathcal{D}(\Omega))^{2 \times 2}$, then (2.17) leads to

$$
\frac{1}{2 \mu}(\sigma, \tau)+(\tau, \omega)+(\operatorname{div}(\tau), u)=0, \quad \forall \tau \in(\mathcal{D}(\Omega))^{2 \times 2} .
$$

Restricting ourselves to symmetric $\tau$ and using the Green formula, we get

$$
\left\langle(2 \mu)^{-1} \sigma-\varepsilon(u), \tau\right\rangle=0, \quad \forall \tau \in(\mathcal{D}(\Omega))^{2 \times 2} \quad \text { symmetric. }
$$

By (2.18) with $v=0, \varphi=0$ and $\eta \in\left(L^{r_{1}}(\Omega)\right)^{2 \times 2}$ such that $\eta+\eta^{\top}=0$, we deduce that $(\sigma, \eta)=0$. Hence $\sigma$ must be symmetric. We therefore deduce from $(2.23)$ that $\sigma=2 \mu \varepsilon(u)$.

Similarly, taking the antisymmetric tensor $\tau$ in $(2.22)$, we deduce that $\omega=(\nabla u-$ $\left.(\nabla u)^{\top}\right) / 2$.

Since $\varepsilon(u) \in\left(L^{r_{1}}(\Omega)\right)^{2 \times 2}$ and $\nabla u-(\nabla u)^{\top} \in\left(L^{r_{1}}(\Omega)\right)^{2 \times 2}$, we see that $\nabla u \in\left(L^{r_{1}}(\Omega)\right)^{2 \times 2}$. As $r_{1}>2$ and $r_{2}>2$, we deduce that $u \in\left(H^{1}(\Omega)\right)^{2}$.

In $(2.17)$ we now take $\underline{\tau}=(\tau, 0)$ with $\tau \in\left(C^{\infty}(\bar{\Omega})\right)^{2 \times 2}$. This yields

$$
(2 \mu)^{-1}(\sigma, \tau)+(\tau, \omega)+(\operatorname{div}(\tau), u)+\langle\tau \cdot n, \xi\rangle_{\Gamma_{N}}=0 .
$$

Applying the Green formula (2.11), this identity is equivalent to

$$
(\tau, \omega)+\langle\tau \cdot n, u\rangle_{\Gamma}+\langle\tau \cdot n, \xi\rangle_{\Gamma_{N}}-\frac{1}{2}(\operatorname{as}(\tau), \text { curl } u)=0 .
$$


Setting $\beta=\left\{\begin{array}{ll}u+\xi & \text { in } \Gamma_{N}, \\ u & \text { in } \Gamma_{D},\end{array}\right.$ the above identity can be written as

$$
(\tau, \omega)+\langle\tau \cdot n, \beta\rangle_{\Gamma}-\frac{1}{2}(\operatorname{as}(\tau), \operatorname{curl} u)=0 .
$$

As $\omega=\left[\nabla u-(\nabla u)^{\top}\right] / 2$, we readily see that $(\tau, \omega)=(\operatorname{as}(\tau)$, curl $u) / 2$ and therefore $\langle\tau \cdot n, \beta\rangle_{\Gamma}=0$. Using a similar procedure as in [25, p. 329], we deduce that $\beta=0$ on $\Gamma$, i.e.,

$$
u= \begin{cases}-\xi & \text { on } \Gamma_{N} \\ 0 & \text { on } \Gamma_{D}\end{cases}
$$

This shows that $\xi=-u_{\mid \Gamma_{N}}$ and that $u \in\left(H_{0, \Gamma_{D}}^{1}(\Omega)\right)^{2}$.

Finally, in (2.18) taking $v=0$ and recalling that $(\sigma, \eta)=0$, we get

$$
\langle(\sigma-p \delta) \cdot n, \varphi\rangle=\langle g, \varphi\rangle_{\Gamma_{N}}, \quad \forall \varphi \in\left(H_{00}^{\frac{1}{2}}\left(\Gamma_{N}\right)\right)^{2}
$$

which means that

$$
(\sigma-p \delta) \cdot n=g
$$

Now let us show that (2.3) holds. In (2.18), we take $v \in(\mathcal{D}(\Omega))^{2}$ and let $\eta=0, \varphi=0$. We have

$$
-\frac{1}{2 \mu}(\sigma \cdot u, v)-(\omega \cdot u, v)+(\operatorname{div}(\sigma-p \delta), v)=-(f, v), \quad \forall v \in(\mathcal{D}(\Omega))^{2} .
$$

Using (2.12) and (2.13), this yields

$$
-((u \cdot \nabla) u, v)+(\operatorname{div}(\sigma-p \delta), v)=-(f, v), \forall v \in(\mathcal{D}(\Omega))^{2} .
$$

This means that

$$
-(u \cdot \nabla) u+\operatorname{div}(\sigma-p \delta)=-f \text { in }\left(\mathcal{D}^{\prime}(\Omega)\right)^{2} .
$$

As $\underline{\sigma} \in \Sigma$, we obtain $\operatorname{div}(\sigma-p \delta) \in\left(L^{t}(\Omega)\right)^{2}$. have

Let us now take $v \in\left(H_{0, \Gamma_{D}}^{1}(\Omega)\right)^{2} \cap\left(W^{1, t^{\prime}}(\Omega)\right)^{2}$ with $1 / t^{\prime}+1 / t=1$. In view of $(2.25)$, we

$$
((u \cdot \nabla) u, v)-(\operatorname{div}(\sigma-p \delta), v)=(f, v) .
$$

Applying the Green formula (2.11), this identity becomes

$$
(\sigma-p \delta, \varepsilon(v))-\langle(\sigma-p \delta) \cdot n, v\rangle_{\Gamma_{N}}+((u \cdot \nabla) u, v)=(f, v) .
$$

Using (2.24), this identity leads to

$$
((\sigma-p \delta), \varepsilon(v))+((u \cdot \nabla) u, v)=(f, v)+\langle g, v\rangle_{\Gamma_{N}},
$$

i.e.,

$$
\int_{\Omega}(2 \mu \varepsilon(u)-p \delta): \varepsilon(v) d x+\int_{\Omega}[(u \cdot \nabla) u] \cdot v d x=\int_{\Omega} f v d x+\langle g, v\rangle_{\Gamma_{N}},
$$

for all $v \in\left(H_{0, \Gamma_{D}}^{1}(\Omega)\right)^{2} \cap\left(W^{1, t^{\prime}}(\Omega)\right)^{2}$. Since $\left(H_{0, \Gamma_{D}}^{1}(\Omega)\right)^{2} \cap\left(W^{1, t^{\prime}}(\Omega)\right)^{2}$ is dense in $\left(H_{0, \Gamma_{D}}^{1}(\Omega)\right)^{2}$, (2.26) remains true for all $v \in\left(H_{0, \Gamma_{D}}^{1}(\Omega)\right)^{2}$. This proves $(2.3)$.

It remains to prove $(2.4)$. To this end, we take $\underline{\tau}=(0, q)$ in $(2.17)$ with $q \in \mathcal{D}(\Omega)$ to get $-(\operatorname{div}(q \delta), u)=0, \forall q \in \mathcal{D}(\Omega)$. This is equivalent to

$$
\int_{\Omega} q \operatorname{div} u d x=0, \quad \forall q \in \mathcal{D}(\Omega)
$$

which implies (2.4). 


\section{The Stokes and Navier — Stokes problems}

3.1. The Stokes problem. For $t^{\prime}=2 /(1+\alpha)<2$, let the operator $S$ be defined by

$$
S:\left(L^{t^{\prime}}(\Omega)\right)^{2} \times\left(H^{1 / 2}\left(\Gamma_{N}\right)\right)^{2} \rightarrow \Sigma \times M, \quad\left(f^{*}, g^{*}\right) \rightarrow S\left(f^{*}, g^{*}\right)=\left(\underline{\sigma}^{*}, \underline{u}^{*}\right),
$$

where $\left(\underline{\sigma}^{*}, \underline{u}^{*}\right)=\left(\left(\sigma^{*}, p^{*}\right),\left(u^{*}, \omega^{*}, \xi^{*}\right)\right) \in \Sigma \times M$ is the solution of the problem

$$
\begin{gathered}
\frac{1}{2 \mu}\left(\sigma^{*}, \tau\right)+\left(\tau, \omega^{*}\right)+\left(\operatorname{div}(\tau-q \delta), u^{*}\right)+\left\langle(\tau-q \delta) \cdot n, \xi^{*}\right\rangle_{\Gamma_{N}}=0, \quad \forall \underline{\tau}=(\tau, q) \in \Sigma, \\
\left(\sigma^{*}, \eta\right)+\left(\operatorname{div}\left(\sigma^{*}-p^{*} \delta\right), v\right)+\left\langle\left(\sigma^{*}-p^{*} \delta\right) \cdot n, \varphi\right\rangle=-\left(f^{*}, v\right)+\left\langle g^{*}, \varphi\right\rangle, \quad \forall \underline{v}=(v, \eta, \varphi) \in M .
\end{gathered}
$$

System (3.1), (3.2) is nothing but the mixed variational formulation of the Stokes problem

$$
\left\{\begin{array}{l}
-2 \mu \operatorname{div} \varepsilon\left(u^{*}\right)+\nabla p^{*}=f^{*} \quad \text { in } \Omega \\
\operatorname{div} u^{*}=0 \quad \text { in } \Omega \\
u^{*}=0 \quad \text { in } \Gamma_{D}, \\
\left(2 \mu \varepsilon\left(u^{*}\right)-p^{*} \delta\right) \cdot n=g^{*} \quad \text { in } \Gamma_{N} .
\end{array}\right.
$$

Theorem 3.1. There exists a unique solution $\left(\underline{\sigma}^{*}, \underline{u}^{*}\right) \in \Sigma \times M$ of the mixed variational formulation (3.1), (3.2). Moreover, if $g^{*}=0$, then $\left\|\left(\underline{\sigma}^{*}, \underline{u}^{*}\right)\right\|_{\Sigma \times M} \leqslant C\left\|f^{*}\right\|_{0, t^{\prime}, \Omega}$, which can be expressed in terms of the operator $S$

$$
\left\|S\left(f^{*}, 0\right)\right\|_{\Sigma \times M} \leqslant C\left\|f^{*}\right\|_{0, t^{\prime}, \Omega},
$$

i.e., the operator $S$ is a continuous operator from $\left(L^{t^{\prime}}(\Omega)\right)^{2} \times\{0\}$ into $\Sigma \times M$.

Proof. Since (3.1), (3.2) is nothing but (2.17), (2.18) without the nonlinear term, the above theorem shows that (3.1), (3.2) is equivalent to the mixed formulation of the Stokes system, i.e., (2.3), (2.4) without the nonlinear term. Since $t^{\prime}<2 /(1+\alpha)$ Corollary 4.2 of [36] guarantees the existence of a unique solution $\left(u^{*}, p^{*}\right)$ of the Stokes system. By the Sobolev embedding theorem we deduce the requested regularity for $\left(\underline{\sigma}^{*}, \underline{u}^{*}\right)$ (see [26]).

Estimate (3.4) with $g^{*}=0$ was proved in [26].

Note that (3.4) implies that

$$
\left\|\left(\underline{\sigma}^{*}, \underline{u}^{*}\right)\right\|_{\hat{\Sigma} \times \hat{M}} \leqslant C\left\|f^{*}\right\|_{0, t^{\prime}, \Omega}
$$

where $\hat{\Sigma}=\left(L^{r}(\Omega)\right)^{2 \times 2} \times L^{r}(\Omega)$,

$$
\hat{M}=\left\{\underline{v}=(v, \eta, \varphi) ; \quad v \in\left[L^{r}(\Omega)\right]^{2}, \quad \eta \in\left[L^{r}(\Omega)\right]^{2 \times 2} \quad \text { with } \eta+\eta^{T}=0, \quad \varphi \in\left(L^{2}\left(\Gamma_{N}\right)\right)^{2}\right\},
$$

with its natural norm

$$
\left\|\left(\underline{\sigma}^{*}, \underline{u}^{*}\right)\right\|_{\hat{\Sigma} \times \hat{M}}:=\left\|\sigma^{*}\right\|_{0, r, \Omega}+\left\|p^{*}\right\|_{0, r, \Omega}+\left\|u^{*}\right\|_{0, r, \Omega}+\left\|\omega^{*}\right\|_{0, r, \Omega}+\left\|\xi^{*}\right\|_{0, \Gamma_{N}}
$$

$r$ being an arbitrary real number chosen but fixed in the open interval $] 2, \min \left\{r_{1}, r_{2}\right\}[$.

The a priori estimate $(3.5)$ can be rewritten as

$$
\left\|S\left(f^{*}, 0\right)\right\|_{\hat{\Sigma} \times \hat{M}} \leqslant C\left\|f^{*}\right\|_{0, t^{\prime}, \Omega},
$$


which means that $S$ is a continuous operator from $\left(L^{t^{\prime}}(\Omega)\right)^{2} \times\{0\}$ into $\hat{\Sigma} \times \hat{M}$.

\subsection{The Navier — Stokes equations. Let us set}

$$
H: \Sigma \times M \rightarrow \Sigma \times M, \quad(\underline{\tau}, \underline{v}) \rightarrow H(\underline{\tau}, \underline{v})=(\underline{\tau}, \underline{v})-S\left(f-(2 \mu)^{-1} \tau \cdot v-\eta \cdot v, g\right) .
$$

Then $(\underline{\sigma}, \underline{u}) \in \Sigma \times M$ is a solution of the Navier — Stokes equations (2.17), (2.18) if and only if $(\underline{\sigma}, \underline{u})$ satisfies

$$
H(\underline{\sigma}, \underline{u})=0 .
$$

In the sequel, we are interested in a non-singular solution of (3.7) in the following sense:

Definition 3.1. $(\underline{\sigma}, \underline{u}) \in \Sigma \times M$ is a nonsingular solution of (3.7) if the Fréchet derivative of $\mathrm{H}$ at the point $(\underline{\sigma}, \underline{u})$

$H^{\prime}(\underline{\sigma}, \underline{u}): \Sigma \times M \rightarrow \Sigma \times M, \quad(\underline{\tau}, \underline{v}) \rightarrow H^{\prime}(\underline{\sigma}, \underline{u})(\underline{\tau}, \underline{v})=(\underline{\tau}, \underline{v})+S\left(\frac{1}{2 \mu}(\sigma \cdot v+\tau \cdot u)+\omega \cdot v+\eta \cdot u, 0\right)$

is an isomorphism.

Accordingly, $(\underline{\sigma}, \underline{u})$ is a nonsingular solution of (3.7) if and only if the linearized mixed formulation of the Navier - Stokes equations at the point $(\underline{\sigma}, \underline{u})=((\sigma, p),(u, \omega, \xi))$

$$
\begin{gathered}
\frac{1}{2 \mu}\left(\sigma^{*}, \tau\right)+\left(\operatorname{div}(\tau-q \delta), u^{*}\right)+\left(\tau, \omega^{*}\right)+\left\langle(\tau-q \delta) \cdot n, \xi^{*}\right\rangle_{\Gamma_{N}}=0, \quad \forall \underline{\tau} \in \Sigma, \\
\left(\operatorname{div}\left(\sigma^{*}-p^{*} \delta\right), v\right)+\left(\sigma^{*}, \eta\right)+\left\langle\left(\sigma^{*}-p^{*} \delta\right) \cdot n, \varphi\right\rangle_{\Gamma_{N}}-\frac{1}{2 \mu}\left(\sigma \cdot u^{*}+\sigma^{*} \cdot u, v\right)-\left(\omega \cdot u^{*}+\omega^{*} \cdot u, v\right)=0, \\
\forall \underline{v}=(v, \eta, \varphi) \in M
\end{gathered}
$$

has the unique solution $\left(\underline{\sigma}^{*}, \underline{u}^{*}\right)=\left(\left(\sigma^{*}, p^{*}\right),\left(u^{*}, \omega^{*}, \xi^{*}\right)\right)=0$ in $\Sigma \times M$, i.e., $H^{\prime}(\underline{\sigma}, \underline{u})$ is injective.

Proposition 3.1. Let us set $\Sigma^{*}=\left(L^{r_{1}}(\Omega)\right)^{2 \times 2} \times L^{r_{1}}(\Omega)$ equipped with its natural norm. Then the operator

$K(\underline{\sigma}, \underline{u}): \Sigma^{*} \times M \rightarrow \Sigma^{*} \times M, \quad(\underline{\tau}=(\tau, q), \underline{v}=(v, \eta, \varphi)) \rightarrow S\left(\frac{1}{2 \mu}(\sigma \cdot v+\tau \cdot u)+\omega \cdot v+\eta \cdot u, 0\right)$

is a linear compact operator. Consequently, $H^{\prime}(\underline{\sigma}, \underline{u})=I+K(\underline{\sigma}, \underline{u})$ is a Fredholm operator of index 0 in the same space.

Proof. The operator $K(\underline{\sigma}, \underline{u})$ does not depend on $\varphi$ and, consequently, the result follows from [26, Proposition 2.5].

Corollary 3.1. If $H^{\prime}(\underline{\sigma}, \underline{u})$ is injective from $\Sigma \times M$ onto itself, then it is an isomorphism in the same space.

Next, we have a property of the operator $K(\underline{\sigma}, \underline{u})$ that follows from [26, Proposition 2.8].

Proposition 3.2. The operator $K(\underline{\sigma}, \underline{u})$ is compact in the Banach space $\widehat{\Sigma} \times \widehat{M}$. 


\section{The discrete problem}

Since the singularities of our problem have a local character, the meshes have to be refined in the neighbourhood of each singular corner. So without loss of generality, we may suppose that only one corner is singular. Morever, by an eventual translation, we may suppose that it is situated at the origin 0 .

Let $\left(\mathcal{T}_{h}\right)_{h>0}$ be a family of triangulations of $\Omega$ satisfying the following conditions: there exist two positive constants $\gamma, \tilde{\gamma}$ such that

1) $h_{K} / \rho_{K} \leqslant \gamma \forall K \in \mathcal{T}_{h}, \forall h>0$;

2) $h_{K} \leqslant \gamma h^{\beta}$ for every $K \in \mathcal{T}_{h}$ such that one of the corners of $K$ is at 0 ;

3) $h_{K} \leqslant \gamma\left(\inf _{x \in K} r^{1-1 / \beta}(x)\right) h$ for every $K \in \mathcal{T}_{h}$ with no corner at 0 ;

4) $h_{K} \geqslant \tilde{\gamma} h^{\beta} \forall K \in \mathcal{T}_{h}, \forall h>0$;

where $h_{K}$ denotes the diameter of $K, \rho_{K}$ denotes the supremum of the diameters of the inscribed circles in $K$, and $\beta \geqslant 1 /(1-\alpha)$.

Remark 4.1. Condition 1) means that the family of triangulations $\left(\mathcal{T}_{h}\right)_{h>0}$ is regular (see [13]). The other conditions hold, in particular, for Raugel's families of triangulations (see [37]).

For a triangle $K \subset \mathbb{R}^{2}$, let $P_{k}, k \geqslant 0$ denote the restrictions of poynomials of total degree $\leqslant k$ to $K$. We set

$$
\mathcal{P}=\left[P_{1}\right]^{2}+\mathbb{R} \operatorname{curl} b_{K},
$$

where $b_{K}$ denotes the "bubble function" defined by $b_{K}(x)=\lambda_{1}(x) \lambda_{2}(x) \lambda_{3}(x)$, with $\lambda_{1}, \lambda_{2}$, $\lambda_{3}$ being the barycentric coordinates on $K$, and curl $b_{K}=\left(\partial b_{K} / \partial x_{2},-\partial b_{K} / \partial x_{1}\right)$.

Let $\left\{\bar{I}_{1}, \cdots, \bar{I}_{m}\right\}$ be the partition of $\Gamma_{N}$ induced by the triangulation, i.e., each $I_{j}=$ $\partial K \cap \bar{\Gamma}_{l}$ for some triangle $K$ of $\mathcal{T}_{h}$ and some $l \in N$, and $\Gamma_{N}=\bigcup_{j=1}^{m} \bar{I}_{j}$ where $I_{j}$ is an open segment.

Due to our construction, each $I_{j}$ is contained on one side of the polygonal line $\Gamma$.

We are now ready to introduce our finite dimensional approximation spaces:

$$
\Sigma_{h}=\left\{\underline{\tau}_{h}=\left(\tau_{h}, q_{h}\right) \in \Sigma:\left.q_{h}\right|_{K} \in P_{1},\left.\quad \tau_{h}\right|_{K} \in[\mathcal{P}]^{2}, \quad \forall K \in \mathcal{T}_{h}\right\}, \quad M_{h}=M_{1 h} \times M_{2 h},
$$

where

$$
\begin{gathered}
M_{1 h}=\left\{\underline{v}_{h}=\left(v_{h}, \eta_{h}\right) \in M:\left.v_{h}\right|_{K} \in\left[P_{0}\right]^{2}, \quad \eta_{h}=\theta_{h} \chi \quad \text { with }\left.\theta_{h}\right|_{K} \in P_{1}, \quad \forall K \in \mathcal{T}_{h}\right\}, \\
M_{2 h}=\left\{\xi_{h} \in\left(H_{00}^{\frac{1}{2}}\left(\Gamma_{N}\right)\right)^{2}: \xi_{h \mid I_{j}} \in P_{1}\left(I_{j}\right), \quad \forall j=1, \cdots, m\right\},
\end{gathered}
$$

where we recall that $\chi=\left[\begin{array}{cc}0 & -1 \\ 1 & 0\end{array}\right]$.

Let us first consider the discrete formulation of the Stokes equations (3.1), (3.2). Namely let $S_{h}$ be the discrete version of $S$, i.e., for $(f, g) \in\left(L^{t^{\prime}}(\Omega)\right)^{2} \times\left(H^{\frac{1}{2}}\left(\Gamma_{N}\right)\right)^{2}, S_{h}(f, g)=$ $\left(\underline{\sigma}_{h}, \underline{u}_{h}\right)=\left(\left(\sigma_{h}, p_{h}\right),\left(u_{h}, \omega_{h}, \xi_{h}\right)\right) \in \Sigma_{h} \times M_{h}$ is the unique solution of

$$
\begin{gathered}
\frac{1}{2 \mu}\left(\sigma_{h}, \tau_{h}\right)+\left(\tau_{h}, \omega_{h}\right)+\left(\operatorname{div}\left(\tau_{h}-q_{h} \delta\right), u_{h}\right)+\left\langle\left(\tau_{h}-q_{h} \delta\right) \cdot n, \xi_{h}\right\rangle_{\Gamma_{N}}=0, \quad \forall\left(\tau_{h}, q_{h}\right) \in \Sigma_{h}, \quad(4.1) \\
\left(\sigma_{h}, \eta_{h}\right)+\left(\operatorname{div}\left(\sigma_{h}-p_{h} \delta\right), v_{h}\right)+\left\langle\left(\left(\sigma_{h}-p_{h} \delta\right) \cdot n, \varphi_{h}\right\rangle_{\Gamma_{N}}=-\left(f, v_{h}\right)+\left\langle g, \varphi_{h}\right\rangle_{\Gamma_{N}}, \quad \forall\left(v_{h}, \eta_{h}, \varphi_{h}\right) \in M_{h} .\right.
\end{gathered}
$$


The existence of a unique solution to this saddle point problem requires the so-called inf-sup condition for the form

$$
b\left(\underline{\tau}_{h}, \underline{v}_{h}\right)=\left(\operatorname{div}\left(\tau_{h}-q_{h} \delta\right), v_{h}\right)+\left(\tau_{h}, \eta_{h}\right)+\left\langle\left(\tau_{h}-q_{h} \delta\right) \cdot n, \varphi_{h}\right\rangle_{\Gamma_{N}},
$$

with $\underline{\tau}_{h}=\left(\tau_{h}, q_{h}\right) \in \Sigma_{h}, \underline{v}_{h}=\left(v_{h}, \eta_{h}, \varphi_{h}\right) \in M_{h}$ and the coerciveness of the form $a\left(\underline{\sigma}_{h}, \underline{\tau}_{h}\right)=$ $(2 \mu)^{-1}\left(\sigma_{h}, \tau_{h}\right)$, with $\underline{\sigma}_{h}=\left(\sigma_{h}, p_{h}\right), \underline{\tau}_{h}=\left(\tau_{h}, q_{h}\right)$, on the space

$$
V_{h}=\left\{\underline{\tau}_{h}=\left(\tau_{h}, q_{h}\right) \in \Sigma_{h}: b\left(\underline{\tau}_{h}, \underline{v}_{h}\right)=0, \quad \forall \underline{v}_{h} \in M_{h}\right\} .
$$

Lemma 4.1. There exists a constant $C>0$ independent of $h$ such that

$$
\sup _{\substack{\tau_{h} \in \Sigma_{h} \\ \underline{\tau}_{h} \neq 0}} \frac{b\left(\underline{\tau}_{h}, \underline{v}_{h}\right)}{\left\|\underline{\tau}_{h}\right\|_{0, \Omega}} \geqslant C\left(\left\|v_{h}\right\|_{0, \Omega}+\left\|\eta_{h}\right\|_{0, \Omega}+\left\|\varphi_{h}\right\|_{0, \Gamma_{N}}\right),
$$

for all $\underline{v}_{h}=\left(v_{h}, \eta_{h}, \varphi_{h}\right) \in M_{h}$.

Proof. We can remark that $\eta_{h}$ can be written as $\eta_{h}=\theta_{h} \chi$, with $\theta_{h} \in L^{r_{1}}(\Omega)$ and $\theta_{h \mid K} \in P_{1}(K)$, for all $K \in \mathcal{T}_{h}$ and that

$$
b\left(\underline{\tau}_{h}, \underline{v}_{h}\right)=\left(\operatorname{div}\left(\tau_{h}-q_{h} \delta\right), v_{h}\right)+\left(a s\left(\tau_{h}\right), \theta_{h}\right)+\left\langle\left(\tau_{h}-q_{h} \delta\right) \cdot n, \varphi_{h}\right\rangle_{\Gamma_{N}} .
$$

Consequently, the inf-sup condition follows from [35, Theorem 3.1].

The above relation (4.3) directly allows to apply Lemma 3.2 of [35] which proved

Lemma 4.2. There exists $C>0$ independent of $h$ such that $a\left(\underline{\tau}_{h}, \underline{\tau}_{h}\right) \geqslant C\left\|\underline{\tau}_{h}\right\|_{0, \Omega}^{2}$, $\forall \tau_{h} \in V_{h}$.

These two lemmas allow to confirm the well-posedness of problem (4.1), (4.2).

Before going on let us recall some usual results from [26, Proposition 3.2] and [35, Proposition 5.1] which we will need in the sequel.

\section{Proposition 4.1. Let}

$$
X_{h}=\left\{v_{h} \in H(\operatorname{div}, \Omega), v_{h \mid K} \in\left[P_{1}\right]^{2}, \forall K \in \mathcal{T}_{h}\right\}, \quad Y_{h}=\left\{q_{h} \in L^{2}(\Omega): q_{h \mid K} \in P_{1}, \forall K \in \mathcal{T}_{h}\right\} .
$$

Let $P_{h}^{1}$ be the $L^{2}$-orthogonal projection from $L^{2}(\Omega)$ onto $Y_{h}$ and let the operator $\rho_{h}$ be defined by $\left.\left(\rho_{h} v\right)\right|_{K}=\rho_{K} v \in\left[P_{1}(K)\right]^{2}$, for all $v \in\left(H^{1, \alpha}(\Omega)\right)^{2}$, where $\rho_{K} v$ is uniquely determined by

$$
\int_{e} \rho_{K} v \cdot n p_{1} d s=\int_{e} v \cdot n p_{1} d s, \quad \forall p_{1} \in P_{1}(e), \quad \forall e \subset \partial K .
$$

Then for all $s \in[2,2 / \alpha[$, there exists $c>0$ independent of $h$ such that

$$
\begin{gathered}
\left\|\rho_{h} v-v\right\|_{0, s, \Omega} \leqslant c h^{1+\beta(-1+2 / s)}|v|_{1, \alpha, \Omega}, \quad \forall v \in\left(H^{1, \alpha}(\Omega)\right)^{2}, \\
\left\|P_{h}^{1} q-q\right\|_{0, s, \Omega} \leqslant c h^{1+\beta(-1+2 / s)}|q|_{1, \alpha, \Omega}, \quad \forall q \in H^{1, \alpha}(\Omega) .
\end{gathered}
$$

Proposition 4.2. There exists an operator

$$
\Pi_{h}: \Sigma \cap\left(\left(H^{1, \alpha}(\Omega)\right)^{2 \times 2} \times H^{1, \alpha}(\Omega)\right) \longrightarrow \Sigma_{h}: \underline{\tau}=(\tau, q) \rightarrow \Pi_{h} \underline{\tau}=\left(\tau_{h}, q_{h}\right),
$$

such that $b\left(\underline{\tau}-\Pi_{h} \underline{\tau}, \underline{v}_{h}\right)=0, \forall \underline{v}_{h} \in M_{h}$ and satisfies for all $\left.s \in\right] 2,2 / \alpha[$

$$
\left\|\underline{\tau}-\Pi_{h} \underline{\tau}\right\|_{0, s, \Omega} \leqslant C h^{1+\beta(-1+2 / s)}|\underline{\tau}|_{1, \alpha, \Omega},
$$

where $|\underline{\tau}|_{1, \alpha, \Omega}=|\tau|_{1, \alpha, \Omega}+|q|_{1, \alpha, \Omega}$ and $C$ is a positive constant independent of $h$. 
We can now turn to the discretization of the Navier - Stokes system. The discrete version of $(2.17),(2.18)$ can be written as follows: Find $\underline{\sigma}_{h}=\left(\sigma_{h}, p_{h}\right) \in \Sigma_{h}$ and $\underline{u}_{h}=$ $\left(u_{h}, \omega_{h}, \xi_{h}\right) \in M_{h}$ such that

$$
\begin{gathered}
\frac{1}{2 \mu}\left(\sigma_{h}, \tau_{h}\right)+\left(\tau_{h}, \omega_{h}\right)+\left(\operatorname{div}\left(\tau_{h}-q_{h} \delta\right), u_{h}\right)+\left\langle\left(\tau_{h}-q_{h} \delta\right) \cdot n, \xi_{h}\right\rangle_{\Gamma_{N}}=0, \quad \forall \underline{\tau}_{h}=\left(\tau_{h}, q_{h}\right) \in \Sigma_{h}, \\
\left(\sigma_{h}, \eta_{h}\right)+\left(\operatorname{div}\left(\sigma_{h}-p_{h} \delta\right), v_{h}\right)+\left\langle\left(\sigma_{h}-p_{h} \delta\right) \cdot n, \varphi_{h}\right\rangle_{\Gamma_{N}}-\frac{1}{2 \mu}\left(\sigma_{h} \cdot u_{h}, v_{h}\right)-\left(\omega_{h} \cdot u_{h}, v_{h}\right)= \\
-\left(f, v_{h}\right)+\left\langle g, \varphi_{h}\right\rangle_{\Gamma_{N}}, \quad \forall \underline{v}_{h}=\left(v_{h}, \eta_{h}, \varphi_{h}\right) \in M_{h} .
\end{gathered}
$$

Now we define the mapping $H_{h}$ from $\Sigma_{h} \times M_{h}$ into itself by

$$
H_{h}\left(\underline{\tau}_{h}, \underline{v}_{h}\right)=\left(\underline{\tau}_{h}, \underline{v}_{h}\right)-S_{h}\left(f-(2 \mu)^{-1} \tau_{h} \cdot v_{h}-\eta_{h} \cdot v_{h}, g\right) .
$$

We then see that the discrete problem (4.7), (4.8) can be written as:

$$
\text { Find }\left(\underline{\sigma}_{h}, \underline{u}_{h}\right) \in \Sigma_{h} \times M_{h} \text { such that } H_{h}\left(\underline{\sigma}_{h}, \underline{u}_{h}\right)=0 \text {. }
$$

We finally introduce the discrete version of the operator $K(\underline{\sigma}, \underline{u})$. Let $P_{h}^{0}$ be the $L^{2}$ orthogonal projection from $\left(L^{2}(\Omega)\right)^{2}$ onto $\left(\prod_{K \in \mathcal{T}_{h}} P_{0}(K)\right)^{2}$ and let $L_{h}^{1}$ be the Lagrange interpolation operator relative to the partition $\left\{\bar{I}_{1}, \cdots, \bar{I}_{m}\right\}$ of $\Gamma_{N}$, i.e., for $\xi \in\left(H_{00}^{\frac{1}{2}}\left(\Gamma_{N}\right)\right)^{2} \cap$ $\left(C\left(\bar{\Gamma}_{N}\right)\right)^{2}$

$$
L_{h}^{1} \xi=\sum_{i=1}^{m-1} \xi\left(v_{i}\right) \lambda_{v_{i}}
$$

where the node $v_{i}$ is equal to $\bar{I}_{i} \cap \bar{I}_{i+1}$ and $\lambda_{v}$ is the standard hat function.

Now for $(\underline{\sigma}, \underline{u})=((\sigma, p),(u, \omega, \xi)) \in \Sigma \times M$, we set $\left(\underline{\sigma}_{h}^{*}, \underline{u}_{h}^{*}\right)=\left(\Pi_{h} \underline{\sigma}, P_{h} \underline{u}\right)$, where $P_{h} \underline{u}=$ $\left(P_{h}^{0} u,\left(P_{h}^{1} \theta\right) \chi, L_{h}^{1} \xi\right)$, with $\omega=\theta \chi$. Then the operator $K_{h}\left(\underline{\sigma}_{h}^{*}, \underline{u}_{h}^{*}\right)$ is defined by

$$
K_{h}\left(\underline{\sigma}_{h}^{*}, \underline{u}_{h}^{*}\right)(\underline{\tau}, \underline{v})=S_{h}\left((2 \mu)^{-1}\left(\sigma_{h}^{*} \cdot v+\tau \cdot u_{h}^{*}\right)+\omega_{h}^{*} \cdot v+\eta \cdot u_{h}^{*}, 0\right),
$$

for all $(\underline{\tau}, \underline{v})=((\tau, q),(v, \eta, \varphi)) \in \hat{\Sigma} \times \hat{M}$. For shortness, we will write $K_{h}\left(\underline{\sigma}_{h}^{*}, \underline{u}_{h}^{*}\right)=K_{h}$ and $K(\underline{\sigma}, \underline{u})=K$.

Remark 4.2. Similarly to (4.4), (4.5), we have for all $s \in[2,2 / \alpha[$

$$
\left\|P_{h}^{0} v-v\right\|_{0, s, \Omega} \leqslant C h^{1+\beta(-1+2 / s)}|v|_{1, \alpha, \Omega}, \quad \forall v \in\left(H^{1, \alpha}(\Omega)\right)^{2} .
$$

Furthermore we have (see, for instance, [28, Theorem 8.4.1.6])

$$
\left\|L_{h}^{1} v_{\mid \Gamma_{N}}-v_{\mid \Gamma_{N}}\right\|_{0, \Gamma_{N}} \leqslant C h|v|_{2, \alpha, \Omega}, \quad \forall v \in\left(H^{2, \alpha}(\Omega)\right)^{2} \cap\left(H_{0, \Gamma_{D}}^{1}(\Omega)\right)^{2} .
$$

Note further that $h \leqslant C h^{1+\beta(-1+2 / s)}$, since $s \geqslant 2$.

\section{Error estimates}

Lemma 5.1. Let $f \in\left(L^{2}(\Omega)\right)^{2}$ and $g \in\left(H^{1 / 2}\left(\Gamma_{N}\right)\right)^{2}$, then the following estimate holds:

$$
\left\|\left(S_{h}-S\right)(f, g)\right\|_{\widehat{\Sigma} \times \widehat{M}} \leqslant C h^{1+\beta(-1+2 / r)}\|S(f, g)\|_{\alpha, \Omega},
$$

where we set

$$
\|S(f, g)\|_{\alpha, \Omega}=\|\sigma\|_{1, \alpha, \Omega}+\|p\|_{1, \alpha, \Omega}+\|u\|_{2, \alpha, \Omega}+\|\omega\|_{1, \alpha, \Omega},
$$

when $S(f, g)=((\sigma, p),(u, \omega, \xi))$. 
Proof. By definition, $S(f, g)=(\underline{\sigma}, \underline{u})=((\sigma, p),(u, \omega, \xi))$ is a solution of the Stokes problem (3.1), (3.2) and $S_{h}(f, g)=\left(\underline{\sigma}_{h}, \underline{u}_{h}\right)=\left(\left(\sigma_{h}, p_{h}\right),\left(u_{h}, \omega_{h}, \xi_{h}\right)\right)$ is a solution of the discrete Stokes problem (4.1), (4.2). Let us set $\left(\sigma_{h}^{*}, p_{h}^{*}\right)=\Pi_{h}(\sigma, p)$ and $\left(u_{h}^{*}, \omega_{h}^{*}, \xi_{h}^{*}\right)=P_{h}(u, w, \xi)=$ $\left(P_{h}^{0} u,\left(P_{h}^{1} \theta\right) \chi, L_{h}^{1} \xi\right)$ with $\omega=\theta \chi$. Then using the theory of mixed finite element methods, we have

$$
\begin{gathered}
\left\|\sigma_{h}^{*}-\sigma_{h}\right\|_{0, \Omega}+\left\|p_{h}^{*}-p_{h}\right\|_{0, \Omega}+\left\|u_{h}^{*}-u_{h}\right\|_{0, \Omega}+\left\|\omega_{h}^{*}-\omega_{h}\right\|_{0, \Omega}+\left\|\xi_{h}^{*}-\xi_{h}\right\|_{0, \Gamma_{N}} \leqslant \\
C\left(\left\|\sigma-\sigma_{h}^{*}\right\|_{0, \Omega}+\left\|p-p_{h}^{*}\right\|_{0, \Omega}+\left\|\omega-\omega_{h}^{*}\right\|_{0, \Omega}\right) .
\end{gathered}
$$

This last inequality with (4.5) and (4.6) yields

$$
\begin{gathered}
\left\|\sigma_{h}^{*}-\sigma_{h}\right\|_{0, \Omega}+\left\|p_{h}^{*}-p_{h}\right\|_{0, \Omega}+\left\|u_{h}^{*}-u_{h}\right\|_{0, \Omega}+\left\|\omega_{h}^{*}-\omega_{h}\right\|_{0, \Omega}+\left\|\xi_{h}^{*}-\xi_{h}\right\|_{0, \Gamma_{N}} \leqslant \\
C h\left(|\sigma|_{1, \alpha, \Omega}+|p|_{1, \alpha, \Omega}+|\omega|_{1, \alpha, \Omega}\right) .
\end{gathered}
$$

Now, using the inverse inequality (owing to 4 ) for $\left.\left(\mathcal{T}_{h}\right)\right)\left\|\psi_{h}\right\|_{0, r, \Omega} \leqslant C h^{\beta(-1+2 / r)}\left\|\psi_{h}\right\|_{0, \Omega}$, this estimate leads to

$$
\begin{gathered}
\left\|\sigma_{h}^{*}-\sigma_{h}\right\|_{0, r, \Omega}+\left\|p_{h}^{*}-p_{h}\right\|_{0, r, \Omega}+\left\|u_{h}^{*}-u_{h}\right\|_{0, r, \Omega}+\left\|\omega_{h}^{*}-\omega_{h}\right\|_{0, r, \Omega}+\left\|\xi_{h}^{*}-\xi_{h}\right\|_{0, \Gamma_{N}} \leqslant \\
C h^{1+\beta(-1+2 / r)}\left(|\sigma|_{1, \alpha, \Omega}+|p|_{1, \alpha, \Omega}+|\omega|_{1, \alpha, \Omega}\right) .
\end{gathered}
$$

Therefore, using (4.5), (4.6), (4.10), (4.11), (5.2) and the triangle inequality, we obtain estimate (5.1).

Next, we also need the following lemma proved as in [26, Corollary 4.3].

Lemma 5.2. Assume that $f \in\left(L^{t^{\prime}}(\Omega)\right)^{2}$, where we recall that $t^{\prime}=2 /(1+\alpha)$. Then for all $r$ such that

$$
2<r<\min \left\{r_{1}, r_{2}, \frac{2 \beta}{\beta-(1-\alpha)}\right\} \text {, }
$$

we have

$$
\left\|\left(S_{h}-S\right)(f, 0)\right\|_{\widehat{\Sigma} \times \widehat{M}} \leqslant C h^{\epsilon}\|f\|_{0, t^{\prime}, \Omega}
$$

for some $\epsilon>0$.

From now on, we fix

$$
\beta=\frac{2}{2-\alpha r}
$$

Lemma 5.3. Assume that $(\underline{\sigma}, \underline{u})$ is a nonsingular solution of (3.7). Then, for all $r$ such that

$$
2<r<\min \left\{r_{1}, r_{2}, \frac{2(2-\alpha)}{1+\alpha(1-\alpha)}\right\} \text {, }
$$

we have $\lim _{h \rightarrow 0}\left\|K-K_{h}\right\|_{\mathcal{L}(\widehat{\Sigma} \times \widehat{M})}=0$.

Proof. We simply remark that

$$
\begin{gathered}
\left(K-K_{h}\right)(\underline{\tau}, \underline{v})=S\left(\frac{1}{2 \mu}(\sigma \cdot v+\tau \cdot u)+\omega \cdot v+\eta \cdot u, 0\right)-S_{h}\left(\frac{1}{2 \mu}\left(\sigma_{h}^{*} \cdot v+\tau \cdot u_{h}^{*}\right)+\omega_{h}^{*} \cdot v+\eta \cdot u_{h}^{*}, 0\right)= \\
\frac{1}{2 \mu} S\left(\left(\sigma-\sigma_{h}^{*}\right) \cdot v, 0\right)+\frac{1}{2 \mu} S\left(\tau \cdot\left(u-u_{h}^{*}\right), 0\right)+S\left(\left(\omega-\omega_{h}^{*}\right) \cdot v, 0\right)+S\left(\eta \cdot\left(u-u_{h}^{*}\right), 0\right)+
\end{gathered}
$$




$$
\frac{1}{2 \mu}\left(S-S_{h}\right)\left(\sigma_{h}^{*} \cdot v, 0\right)+\frac{1}{2 \mu}\left(S-S_{h}\right)\left(\tau \cdot u_{h}^{*}, 0\right)+\left(S-S_{h}\right)\left(\omega_{h}^{*} \cdot v, 0\right)+\left(S-S_{h}\right)\left(\eta \cdot u_{h}^{*}, 0\right) .
$$

Note that $K-K_{h}$ does not depend on the variable $\varphi$ (corresponding to the Lagrange multiplier) and is therefore almost identical to the one from [26], with the exception of the component corresponding to the Lagrange multiplier. The conclusion then follows from Lemma 5.2, property (3.6) and estimates (4.5), (4.6), and (4.10) (compare to [26, Lemma $4.4])$.

If $(\underline{\sigma}, \underline{u})$ is a nonsingular solution of $(3.7)$, then $I+K$ is an isomorphism, and by a classical perturbation argument, the above result yields

Lemma 5.4. Assume that $(\underline{\sigma}, \underline{u})$ is a nonsingular solution of (3.7). Then for a sufficiently small $h$, the operator $I+K_{h}$ is an isomorphism from $\widehat{\Sigma} \times \widehat{M}$ into itself. Moreover, the operator $\left(I+K_{h}\right)^{-1}$ maps $\Sigma_{h} \times M_{h}$ into itself, and its norm is bounded independently of $h$.

Lemma 5.5. Assume that $(\underline{\sigma}, \underline{u})$ is a nonsingular solution of (3.7) and

$$
2<r<\min \left\{r_{1}, r_{2}, \frac{2(2-\alpha)}{1+\alpha(1-\alpha)}\right\} \text {. }
$$

Then there exists $C>0$ such that

$$
\left\|H_{h}\left(\underline{\sigma}_{h}^{*}, \underline{u}_{h}^{*}\right)\right\|_{\widehat{\Sigma} \times \widehat{M}} \leqslant C h^{1+\beta(-1+2 / r)}
$$

where we recall that $\underline{\sigma}_{h}^{*}=\left(\sigma_{h}^{*}, p_{h}^{*}\right)=\Pi_{h}(\sigma, p)$ and $\underline{u}_{h}^{*}=\left(u_{h}^{*}, \omega_{h}^{*}, \xi_{h}^{*}\right)=P_{h}(u, \omega, \xi)$.

Proof. By definiton,

$$
H_{h}\left(\underline{\sigma}_{h}^{*}, \underline{u}_{h}^{*}\right)=\left(\underline{\sigma}_{h}^{*}, \underline{u}_{h}^{*}\right)-S_{h}\left(f-(2 \mu)^{-1} \sigma_{h}^{*} \cdot u_{h}^{*}-\omega_{h}^{*} \cdot u_{h}^{*}, g\right),
$$

and

$$
H(\underline{\sigma}, \underline{u})=(\underline{\sigma}, \underline{u})-S\left(f-(2 \mu)^{-1} \sigma \cdot u-\omega \cdot u, g\right)=0 .
$$

Subtracting these two identities, we obtain:

$H_{h}\left(\underline{\sigma}_{h}^{*}, \underline{u}_{h}^{*}\right)=\left(\underline{\sigma}_{h}^{*}-\underline{\sigma}, \underline{u}_{h}^{*}-\underline{u}\right)+\left(S-S_{h}\right)\left(f-\frac{1}{2 \mu} \sigma \cdot u-\omega \cdot u, g\right)+S_{h}\left(\frac{1}{2 \mu}\left(\sigma_{h}^{*} \cdot u_{h}^{*}-\sigma \cdot u\right)+\omega_{h}^{*} \cdot u_{h}^{*}-\omega \cdot u, 0\right)$.

From (4.5), (4.6), (4.10), (4.11) and (5.1) we have

$$
\begin{gathered}
\left\|\left(\underline{\sigma}_{h}^{*}-\underline{\sigma}, \underline{u}_{h}^{*}-\underline{u}\right)\right\|_{\widehat{\Sigma} \times \widehat{M}} \leqslant C h^{1+\beta(-1+2 / r)}, \\
\left\|\left(S-S_{h}\right)\left(f-(2 \mu)^{-1} \sigma \cdot u-\omega \cdot u, g\right)\right\|_{\widehat{\Sigma} \times \widehat{M}} \leqslant C h^{1+\beta(-1+2 / r)},
\end{gathered}
$$

Moreover, using Lemma 5.2, property (3.6) and estimates (4.5), (4.6) and (4.10), we show that

$$
\left\|S_{h}\left(\frac{1}{2 \mu}\left(\sigma_{h}^{*} \cdot u_{h}^{*}-\sigma \cdot u\right)+\omega_{h}^{*} \cdot u_{h}^{*}-\omega \cdot u, 0\right)\right\|_{\widehat{\Sigma} \times \widehat{M}} \leqslant C h^{1+\beta\left(\frac{2}{r}-1\right)} .
$$

This estimate and (5.6) - (5.8) lead to (5.5).

We are now ready to prove the error estimate for the nonsingular solution of (3.7) or (2.17), (2.18). 
Theorem 5.1. If $(\underline{\sigma}, \underline{u})$ is a nonsingular solution of (3.7), then for $h$ small enough, problem (4.9) has at least one solution $\left(\underline{\sigma}_{h}, \underline{u}_{h}\right)$ such that

$$
\left\|(\underline{\sigma}, \underline{u})-\left(\underline{\sigma}_{h}, \underline{u}_{h}\right)\right\|_{\widehat{\Sigma} \times \widehat{M}} \leqslant C h^{(8-2 r-2 \alpha r) /\left(2 r-\alpha r^{2}\right)},
$$

where

$$
2<r<\min \left\{r_{1}, r_{2}, \frac{2(1-\alpha)}{1+\alpha(1-\alpha)}\right\} .
$$

Proof. We define the mapping $\mathcal{R}$ from $\Sigma_{h} \times M_{h}$ into itself by

$$
\mathcal{R}\left(\underline{\tau}_{h}, \underline{v}_{h}\right)=\left(\underline{\tau}_{h}, \underline{v}_{h}\right)-\left(I+K_{h}\right)^{-1} H_{h}\left(\underline{\tau}_{h}, \underline{v}_{h}\right)
$$

and prove that it has a fixed point in the neighborhood of $\left(\underline{\sigma}_{h}^{*}, \underline{u}_{h}^{*}\right)$ where we recall that $\underline{\sigma}_{h}^{*}=\left(\sigma_{h}^{*}, p_{h}^{*}\right)=\Pi_{h}(\sigma, p)$ and $\underline{u}_{h}^{*}=\left(u_{h}^{*}, \omega_{h}^{*}, \xi_{h}^{*}\right)=P_{h}(u, \omega, \xi)$.

Using Lemma 5.4, we can write

$$
\mathcal{R}\left(\underline{\tau}_{h}, \underline{v}_{h}\right)-\left(\underline{\sigma}_{h}^{*}, \underline{u}_{h}^{*}\right)=\left(I+K_{h}\right)^{-1}\left(I+K_{h}\right)\left(\mathcal{R}\left(\underline{\tau}_{h}, \underline{v}_{h}\right)-\left(\underline{\sigma}_{h}^{*}, \underline{u}_{h}^{*}\right)\right)
$$

and then

$$
\left\|\mathcal{R}\left(\underline{\tau}_{h}, \underline{v}_{h}\right)-\left(\underline{\sigma}_{h}^{*}, \underline{u}_{h}^{*}\right)\right\|_{\widehat{\Sigma} \times \widehat{M}} \leqslant C\left\|\left(I+K_{h}\right)\left(\mathcal{R}\left(\underline{\tau}_{h}, \underline{v}_{h}\right)-\left(\underline{\sigma}_{h}^{*}, \underline{u}_{h}^{*}\right)\right)\right\|_{\widehat{\Sigma} \times \widehat{M}}
$$

On the other hand, we can write

$$
\begin{gathered}
\left(I+K_{h}\right)\left(\mathcal{R}\left(\underline{\tau}_{h}, \underline{v}_{h}\right)-\left(\underline{\sigma}_{h}^{*}, \underline{u}_{h}^{*}\right)\right)=\left(I+K_{h}\right)\left[\left(\underline{\tau}_{h}, \underline{v}_{h}\right)-\left(I+K_{h}\right)^{-1} H_{h}\left(\underline{\tau}_{h}, \underline{v}_{h}\right)-\left(\underline{\sigma}_{h}^{*}, \underline{u}_{h}^{*}\right)\right]= \\
\left(I+K_{h}\right)\left[\left(\underline{\tau}_{h}, \underline{v}_{h}\right)-\left(\underline{\sigma}_{h}^{*}, \underline{u}_{h}^{*}\right)\right]-H_{h}\left(\underline{\tau}_{h}, \underline{v}_{h}\right)= \\
H_{h}\left(\underline{\sigma}_{h}^{*}, \underline{u}_{h}^{*}\right)-H_{h}\left(\underline{\tau}_{h}, \underline{v}_{h}\right)+\left(I+K_{h}\right)\left[\left(\underline{\tau}_{h}, \underline{v}_{h}\right)-\left(\underline{\sigma}_{h}^{*}, \underline{u}_{h}^{*}\right)\right]-H_{h}\left(\underline{\sigma}_{h}^{*}, \underline{u}_{h}^{*}\right)= \\
-S_{h}\left((2 \mu)^{-1}\left(\sigma_{h}^{*}-\tau_{h}\right) \cdot\left(u_{h}^{*}-v_{h}\right)+\left(\omega_{h}^{*}-\eta_{h}\right) \cdot\left(u_{h}^{*}-v_{h}\right), 0\right)-H_{h}\left(\underline{\sigma}_{h}^{*}, \underline{u}_{h}^{*}\right) .
\end{gathered}
$$

Now (3.6) and (5.3) yield $\left\|S_{h}(f, 0)\right\|_{\widehat{\Sigma} \times \widehat{M}} \leqslant C\|f\|_{0, t^{\prime}, \Omega}, \forall f \in\left(L^{t^{\prime}}(\Omega)\right)^{2}$. This estimate and the inverse inequality $\left\|v_{h}\right\|_{0, s, \Omega} \leqslant C h^{\beta(2 / s-2 / r)}\left\|v_{h}\right\|_{0, r, \Omega}$ allow to write

$$
\begin{gathered}
\left\|S_{h}\left(\left(\sigma_{h}^{*}-\tau_{h}\right) \cdot\left(u_{h}^{*}-v_{h}\right), 0\right)\right\|_{\widehat{\Sigma} \times \widehat{M}} \leqslant C\left\|\left(\sigma_{h}^{*}-\tau_{h}\right) \cdot\left(u_{h}^{*}-v_{h}\right)\right\|_{0, t^{\prime}, \Omega} \leqslant C\left\|\sigma_{h}^{*}-\tau_{h}\right\|_{0, r, \Omega}\left\|u_{h}^{*}-v_{h}\right\|_{0, s, \Omega} \leqslant \\
C h^{\beta(2 / s-2 / r)}\left\|\sigma_{h}^{*}-\tau_{h}\right\|_{0, r, \Omega}\left\|u_{h}^{*}-v_{h}\right\|_{0, r, \Omega} \leqslant C h^{\beta(2 / s-2 / r)}\left(\left\|\sigma_{h}^{*}-\tau_{h}\right\|_{0, r, \Omega}+\left\|u_{h}^{*}-v_{h}\right\|_{0, r, \Omega}\right)^{2},
\end{gathered}
$$

where $1 / s=1 / t^{\prime}-1 / r=(1+\alpha) / 2-1 / r$.

Similar arguments yield

$$
\left\|S_{h}\left(\left(\omega_{h}^{*}-\eta_{h}\right) \cdot\left(u_{h}^{*}-v_{h}\right), g\right)\right\|_{\widehat{\Sigma} \times \widehat{M}} \leqslant C h^{\beta(2 / s-2 / r)}\left(\left\|\omega_{h}^{*}-\eta_{h}\right\|_{0, r, \Omega}+\left\|u_{h}^{*}-v_{h}\right\|_{0, r, \Omega}\right)^{2} .
$$

These two estimates and (5.10), (5.11) and (5.5) lead to

$$
\left\|\mathcal{R}\left(\underline{\tau}_{h}, \underline{v}_{h}\right)-\left(\underline{\sigma}_{h}^{*}, \underline{u}_{h}^{*}\right)\right\|_{\widehat{\Sigma} \times \widehat{M}} \leqslant C_{1} h^{\beta(1+\alpha-4 / r)}\left\|\left(\underline{\tau}_{h}, \underline{v}_{h}\right)-\left(\underline{\sigma}_{h}^{*}, \underline{u}_{h}^{*}\right)\right\|_{\widehat{\Sigma} \times \widehat{M}}^{2}+C_{2} h^{1+\beta(-1+2 / r)} .
$$

Consequently, if

$$
\left\|\left(\underline{\tau}_{h}, \underline{v}_{h}\right)-\left(\underline{\sigma}_{h}^{*}, \underline{u}_{h}^{*}\right)\right\|_{\widehat{\Sigma} \times \widehat{M}}^{2} \leqslant \rho(h)
$$

with $\rho(h)$ satisfying

$$
C_{1} h^{\beta(1+\alpha-4 / r)} \rho(h)^{2}+C_{2} h^{1+\beta(-1+2 / r)} \leqslant \rho(h),
$$


we will have

$$
\left\|\mathcal{R}\left(\underline{\tau}_{h}, \underline{v}_{h}\right)-\left(\underline{\sigma}_{h}^{*}, \underline{u}_{h}^{*}\right)\right\|_{\widehat{\Sigma} \times \widehat{M}} \leqslant \rho(h) .
$$

Next, owing to definition (5.4) of $\beta$ and the fact that $r>2$, if $h$ is small enough, the equation

$$
C_{1} h^{\beta(1+\alpha-4 / r)} \rho^{2}-\rho+C_{2} h^{1+\beta(-1+2 / r)}=0
$$

has two real roots, whose mean value is $\rho_{0}(h)=\left(2 C_{1}\right)^{-1} h^{\beta(-1-\alpha+4 / r)}$. Therefore, $\rho(h)=\rho_{0}(h)$ satisfies (5.13) and then (5.12) implies (5.14), in other words, $\mathcal{R}$ maps the ball

$$
\mathcal{B}_{h}=\left\{\left(\underline{\sigma}_{h}, \underline{u}_{h}\right) \in \Sigma_{h} \times M_{h}:\left\|\left(\underline{\tau}_{h}, \underline{v}_{h}\right)-\left(\underline{\sigma}_{h}^{*}, \underline{u}_{h}^{*}\right)\right\|_{\widehat{\Sigma} \times \widehat{M}} \leqslant \rho_{0}(h)\right\}
$$

into itself. As a consequence, $\mathcal{R}$ has at least one fixed point $\left(\underline{\sigma}_{h}, \underline{u}_{h}\right)$ in the ball $\mathcal{B}_{h}$ and such a fixed point is a solution of $H_{h}\left(\underline{\sigma}_{h}, \underline{u}_{h}\right)=0$.

Since $\left(\underline{\sigma}_{h}, \underline{u}_{h}\right) \in \mathcal{B}_{h}, \rho_{0}(h) \leqslant C h^{\beta(-1-\alpha+4 / r)}$ and using (5.7), we have

$$
\left\|(\underline{\sigma}, \underline{u})-\left(\underline{\sigma}_{h}, \underline{u}_{h}\right)\right\|_{\widehat{\Sigma} \times \widehat{M}} \leqslant C h^{\beta(-1-\alpha+4 / r)} .
$$

As $\beta=2 /(2-\alpha r)$, this last estimate leads to the desired result.

Note that estimate (5.9) is quasi-optimal since $(8-2 r-2 \alpha r) /\left(2 r-\alpha r^{2}\right)$ tends to 1 as $r$ goes to 2 .

\section{References}

1. A. Agouzal and J.-M. Thomas, An extension theorem for equilibrium finite element spaces, Japan J. Ind. Applied Math., 13 (1996), pp. 257-266.

2. T. Apel, A.-M. Sändig, J. R. Whiteman, Graded mesh refinement and error estimates for finite element solutions of elliptic boundary value problems in non-smooth domains, Math. Methods Appl. Sci., 19 (1996), pp. 63-85.

3. I. Babuška, Finite element method for elliptic equations for domains with corners, Computing, 6 (1970), pp. 264-273.

4. IBabuska, The finite element methods with Lagrangian multipliers, Numer. Math, 20 (1973), pp. 179-192.

5. I. Babuška and M. R. Rosenzweig, A finite element scheme for domains with corners, Numer. Math., 20 (1972), pp. 1-21.

6. I. Babuška, R. B. Kellogg, and J.Pitkäranta, Direct and inverse error estimates for finite elements with mesh refinements, Numer. Math., 33 (1979), pp.447-471.

7. I. Babuska and G. N. Gatica, On the mixed finite element method with Lagrange multipliers, Numer. Meth. PDE, 19 (2003), pp. 192-210.

8. R. Becker and R. Rannacher, Finite element solution of the incompressible Navier - Stokes equations on anisotropically refined meshes, in Fast solvers for flow problems, Notes on Numerical Fluid Mechanics, Vieweg, Wiesbaden, 49 (1995), pp. 52-62.

9. C. Bernardi and G. Raugel, Méthodes d'éléments finis mixtes pour les équations de Stokes et de Navier - Stokes dans un polygone non convexe, Calcolo, 18 (1981), pp. 255-291.

10. L. Boulaajine, M. Farhoul, and L.Paquet, A posteriori error estimation for the dual mixed finite element method of the elasticity problem in a polygonal domain, Numer. Meth. PDE, 21 (2005), pp.938960.

11. L. Boulaajine, Méthode des éléments finis mixte duale pour les problèmes de l'élasticité et de l'élastodynamique: analyse d'erreur a priori et a posteriori, $\mathrm{PhD}$ thesis, Univ. of Valenciennes, France, July 2006.

12. F. Brezzi and M. Fortin, Mixed and hybrid finite element methods, Springer-Verlag, New-York, 1991.

13. P. G. Ciarlet, The finite element method for elliptic problems, Studies in Math. and its appl., 4, North-Holland, 1978.

14. M. Dauge, Opérateur de Stokes dans des espaces de Sobolev à poids sur des domaines anguleux, Canadian J. Math., 34 (1982), pp. 853-882. 
15. M. Dauge, Elliptic boundary value problems in corner domains. Smoothness and asymptotics of solutions, L. N. in Math., 1341, Springer-Verlag, 1988.

16. M. Dauge, Stationary Stokes and Navier - Stokes systems on two- or three-dimensional domains with corners. Part I: Linearized equations, Siam J. Math. Anal., 20 (1989), PP.74-97.

17. J. Douglas and J.E. Robert, Global estimates for mixed methods for second order elliptic equations, Math. of Computations, 44 (1985), pp. 34-52.

18. H. El Bouzid and S. Nicaise, Refined mixed finite element method for the Stokes problem, in M. Bach, C. Constanda, G. C. Hsiao, A.-M. Sändig, and P. Werner, eds, Analysis, numerics and applications of differential and integral equations, Pitman Research Notes in Mathematics, 379, Longman, Harlow, 1998, pp. $158-162$.

19. M. Farhoul Méthodes d'éléments finis mixtes et volumes finis, Thése, Université de Laval, Québec, 1991.

20. M. Farhloul and M. Fortin, A new mixed finite element for the Stokes and elasticity problems, SIAM J. Numer. Anal., 30 (1993), pp. 971-990.

21. M. Farhloul and H. Manouzi, Analysis of non-singular solutions of a mixed Navier — Stokes formulation, Comput. Methods Appl. Mech. Engrg., 129 (1996), pp. 115-131.

22. M. Farhloul and M. Fortin, Dual hybrid methods for the elasticity and the Stokes problems: A unified approach, Numer. Math., 76 (1997), pp. 419-440.

23. M. Farhoul, S. Nicaise and L. Paquet, A Mixed formulation of Boussinesq equations: analysis of nonsingular solutions, Math. of Computation, 69 (2000), pp. 965-986.

24. M. Farhloul, S. Nicaise and L. Paquet, A refined mixed finite element method for the Boussinesq equations in polygonal domains, IMA J. Numer. Anal., 21 (2001), pp. 525-551.

25. M. Farhoul and L. Paquet, Refined mixed finite element method for the elasticity problem in a polygonal domain, Numer. Meth. PDE, 18 (2002), pp. 323-339.

26. M. Farhoul, S. Nicaise, and L. Paquet, Refined mixed finite element methods of the Navier - Stokes equations with mixed boundary conditions, IMA J. Numer. Anal., submitted, 2005.

27. V. Girault and P. A. Raviart, Finite element methods for Navier — Stokes equations. Theory and algorithms, Springer-Verlag, 1986.

28. P. Grisvard, Elliptic problems in nonsmooth domains, Monographs and Studies in Mathematics, 21, Pitman, Boston, 1985.

29. P. Grisvard, Boundary value problems in plane polygons. Instructions for use (in French), Bulletin de la Direction des Etudes et Recherches EDF, Série C. Mathématique Informatique, 1986, pp. 21-59.

30. P. Grisvard, Singularité en elasticité, Arch. for Rational Mech. Anal., 107 (1989), pp. $157-180$.

31. P. Grisvard, Singularities in boundary value problems, RMA, 22, Masson, Paris, 1992.

32. J. Pitkäranta, Local stability conditions for the Babuska method of Lagrange multipliers, Math. of Computation, 35 (1980), pp. 1113-1129.

33. J. Pitkäranta, The finite element method with Lagrange multipliers for domains with corners, Math. of Computation, 37 (1981), pp. 13-30.

34. S. Nicaise, Polygonal interface problems, Methoden und Verfahren der Mathematischen Physik, bf 39, Peter Lang Verlag, 1993.

35. S. Nicaise, L. Paquet, and Rafilipojaona, Dual mixed finite element methods of the elasticity problem with Lagrange multipliers, J. Comp. Appl. Math., submitted, 2006.

36. M. Orlt and A.-M.Sändig, Regularity of viscous Navier — Stokes flows in nonsmooth domains, in: M. Costabel, M. Dauge and S. Nicaise eds., Boundary value problems and integral equations in nonsmooth domains, Lecture Notes in Pure and Applied Mathematics, 167, Marcel Dekker, 1994, pp. 185-201.

37. G. Raugel, Résolution numérique par une méthode d'éléments finis du problème de Dirichlet pour le Laplacien dans un polygone C. R. Acad. Sci. Paris, Série A, 286 (1978), pp. 791-794.

38. P. A. Raviart and J. M. Thomas, A mixed finite element method for second order elliptic problems, in I. Galligani and E. Magenes eds., Mathematical aspects of Finite Element Methods, Lecture Notes in Mathematics 606, Springer-Verlag, 1977, pp. 292-315.

39. J.E. Roberts and J.M. Thomas, Mixed and hybrid methods, in J.L. Lions and P. G. Ciarlet eds, Handbook of Numerical Analysis, Vol. II, Finite Element Methods (Part 1), North-Holland, Amsterdam, 1991, pp. 523-639. 